Article

\title{
Could Cardiovascular Health Metrics Account for Age and Sex Disparities in Self-Reported Ischemic Heart Disease Prevalence?
}

\author{
Yang Peng * and Zhiqiang Wang \\ Centre for Chronic Disease, School of Clinical Medicine, The University of Queensland, Herston, QLD 4006, \\ Australia; z.wang@uq.edu.au \\ * Correspondence: y.peng@uq.edu.au; Tel.: +61-7-33465149
}

Received: 7 September 2018; Accepted: 17 October 2018; Published: 19 October 2018

\begin{abstract}
The American Heart Association has outlined seven modifiable cardiovascular health $(\mathrm{CVH})$ metrics. However, the sex and age disparities in the association between those CVH metrics and ischemic heart disease (IHD) prevalence are unclear. Our study sought to examine the possible sex and age variations in the association between $\mathrm{CVH}$ metrics and IHD prevalence using an Australian nationally representative survey. We used the core sample of the 2011-2012 Australian Health Survey, and 7499 adults with fasting plasma glucose (FPG) and total cholesterol values were included. We used Poisson regression analysis to measure the associations between individual metrics and IHD prevalence. Our study used both stratification and interaction analyses to compare the magnitude of associations between sex and age groups. Then, we calculated the population attributable fractions to measure the contribution of each metric to IHD prevalence. In addition, we applied logistic regression analysis to examine the influences of ideal CVH metrics number on IHD prevalence and used stratification and interaction analyses. Body mass index, physical activity, blood pressure, and FPG have greater effects on IHD prevalence in young adults compared to older adults. We failed to detect the sex variations in CVH metrics and IHD prevalence. The ideal CVH metrics number was inversely correlated to IHD prevalence and it has similar effects in four subgroups. These CVH metrics do not explain the sex and age disparities in IHD prevalence and the topic need further explorations.
\end{abstract}

Keywords: cardiovascular health; ischemic heart disease; Australian adults

\section{Introduction}

Ischemic heart disease (IHD) is one common type of cardiovascular disease (CVD) and is a significant contributor to deaths worldwide. According to the Global Burden of Disease Study, IHD led to nearly 10 million deaths worldwide in the year 2016, which accounted for $17.3 \%$ of all deaths [1]. There is evidence that a large proportion of IHD burden could be attributable to modifiable factors. A Chinese cohort found that almost half of the IHD cases could be prevented if the participants adhered to six healthy lifestyle factors, including smoking, alcohol consumption, physical activity, dietary pattern, and body mass index (BMI) [2]. In a US study, six lifestyle factors could explain $73 \%$ of coronary heart disease (CHD), another term for IHD, events in a 20-years follow-up period [3].

The American Heart Association (AHA) has outlined seven modifiable metrics [smoking, BMI, physical activity, dietary pattern, total cholesterol (TC), blood pressure (BP), and fasting plasma glucose (FPG)] to define and monitor cardiovascular health (CVH) status in the general population [4]. Some studies have reported that ideal status of those metrics played a crucial role in the reduction of IHD risk $[5,6]$. A few studies have suggested that the strengths of association between modifiable 
factors and IHD risk differed by age and sex $[7,8]$ while the variations were inconclusive due to very limited studies.

In our study, we analysed and compared the strengths of association between CVH metrics and IHD prevalence in sex and age-specific subpopulations using a nationally representative survey in Australia.

\section{Methods}

\subsection{Study Design and Participants}

Details of the study design and participants have been described previously [5]. Briefly, our study used the core sample of 2011-2012 Australian Health Survey, which is a nationally representative health survey of the general Australians. Among 24,910 adults ( $\geq 18$ years), we focused on those with available TC and FPG results, yielding to an overall sample size of 7499. Participants were classified into two age groups: young ( $<60$ years) and older ( $\geq 60$ years).

\subsection{CVH Metrics}

The definitions of the seven CVH metrics were described elsewhere [5]. Subjects with 0-2, 3-4, and 5-7 ideal metrics were regarded as having overall poor, intermediate, and ideal CVH status [9].

\subsection{Outcome Measurement}

The IHD prevalence was self-reported and based on the 10th version of International Classification of Diseases, codes I20-I25. To be more specific, participants were asked if they had been told by a doctor or nurse that they had IHD and they currently have IHD while taking the survey. They would be regarded as positive for IHD if they answered "yes" to both questions.

\subsection{Covariates}

The following variables were adjusted as covariates in our study: age, sex, education attainment, income status, and residence region. Education attainment was categorized as high $(\geq 12$ school years) and low $(<12$ school years). Income status was evaluated by household income and dichotomized as low ( $\leq 50$ th percentile equivalised weekly household income) and high $(>50$ th percentile equivalised weekly household income). Residence region was classified into major cities, inner regional areas and other areas (outer regional and remote).

\subsection{Statistical Analysis}

Firstly, we compared the proportions of IHD, ideal metrics, and covariates between sex and age groups using weighted chi-square tests.

Secondly, we explored the possible sex and age variations in the associations and contributions of individual metrics and IHD prevalence. We calculated crude (unadjusted) and adjusted incidence rate ratios (IRRs) and corresponding $95 \%$ confidence intervals (CIs), using univariate and multivariate Poisson regression analyses, to clarify the association between CVH metrics and IHD prevalence in each sex and age-specific group. We entered the interaction terms between sex/age and metrics in the adjusted models to explore the potential sex/age differences in the association.

Additionally, we calculated adjusted population attributable fractions (PAFs), for each sex and age group, based on the following equation to measure the effects of each metric on IHD reduction. $\mathrm{Pe}$ is the prevalence of unideal metric and Rate Ratios (RRs) were replaced with adjusted IRRs.

$$
P A F=\frac{P e \times(R R-1)}{1+\operatorname{Pe} \times(R R-1)}
$$

Thirdly, we calculated odds ratios (ORs) using logistic regression analyses to explore the relationship between overall CVH status categories and IHD prevalence in sex and age subgroups. 
Interaction terms between sex/age and $\mathrm{CVH}$ categories were included in the adjusted models. Participants with missing values in at least one CVH metrics were not included in the analyses.

To infer results for the in-scope population, we used biomedical weight and group Jackknife method with 60 replicate weights in the CVH metrics and IHD association analyses. All analyses were conducted within the Australian Bureau of Statistics (ABS)'s Remote Access Data Laboratory with Stata 10.0. A two-tailed $p$-value $<0.05$ was used to determine statistical significance.

\section{Results}

\subsection{Baseline Characteristics}

The basic characteristics of the included participants were displayed in Table 1. Overall, weighted $3.3 \%$ participants were positive for IHD prevalence (357/7499). Males have higher IHD prevalence than did the females $(4.2 \%$ versus $2.5 \%, p<0.01)$ and older adults have statistically higher IHD prevalence when compared to young adults $(10.2 \%$ versus $1.1 \%, p<0.01)$. Females had higher proportions of ideal smoking, BMI, dietary pattern, BP, and FPG and lower ideal physical activity proportion and income status than did males. They have similar ideal TC prevalence, education level, and regional distribution. Compared to young adults, older adults had a significantly higher percentage of ideal dietary pattern and lower prevalence of ideal status for the other six metrics. Young adults had higher income and education level and were more likely to reside in major cities as compared to older adults.

Table 1. Baseline characteristics of the included participants.

\begin{tabular}{|c|c|c|c|c|c|c|c|}
\hline Metrics & Status & Males, $n(\%)$ & Females, $n(\%)$ & $p$ & Young, $n(\%)$ & Older, $n(\%)$ & $p$ \\
\hline \multirow[t]{2}{*}{ IHD } & Yes & $214(4.2)$ & $143(2.5)$ & $<0.01$ & $72(1.1)$ & $285(10.2)$ & $<0.01$ \\
\hline & No & $3115(95.8)$ & 4027 (97.5) & & 4751 (98.9) & $2391(89.8)$ & \\
\hline \multirow{2}{*}{ Smoking } & Ideal & $1420(49.8)$ & $2342(61.1)$ & $<0.01$ & $2521(57.7)$ & $1241(49.0)$ & $<0.01$ \\
\hline & Non-ideal & 1909 (50.2) & $1828(38.9)$ & & $2302(42.3)$ & 1435 (51.0) & \\
\hline \multirow[t]{2}{*}{ BMI } & Ideal & $833(32.3)$ & $1562(46.2)$ & $<0.01$ & $1750(43.1)$ & $645(27.2)$ & $<0.01$ \\
\hline & Non-ideal & $2388(67.7)$ & $2344(53.8)$ & & $2843(56.9)$ & $1889(72.8)$ & \\
\hline \multirow[t]{2}{*}{ Physical activity } & Ideal & $945(32.7)$ & 829 (20.9) & $<0.01$ & $1320(30.0)$ & 454 (16.6) & $<0.01$ \\
\hline & Non-ideal & $2382(67.3)$ & 3339 (79.1) & & $3500(70.0)$ & $2221(83.4)$ & \\
\hline \multirow[t]{2}{*}{ Dietary pattern } & Ideal & $93(2.1)$ & $341(7.4)$ & $<0.01$ & $223(4.1)$ & $211(6.9)$ & $<0.01$ \\
\hline & Non-ideal & $3236(97.9)$ & 3829 (92.6) & & $4600(95.9)$ & 2465 (93.1) & \\
\hline \multirow[t]{2}{*}{$\mathrm{TC}$} & Ideal & 1259 (45.1) & $1568(45.8)$ & 0.70 & $2246(52.5)$ & $581(23.9)$ & $<0.01$ \\
\hline & Non-ideal & $2070(54.9)$ & $2602(54.2)$ & & $2577(47.5)$ & $2095(76.1)$ & \\
\hline \multirow[t]{2}{*}{$\mathrm{BP}$} & Ideal & 1047 (36.7) & 1781 (51.6) & $<0.01$ & $2234(51.4)$ & $594(21.9)$ & $<0.01$ \\
\hline & Non-ideal & $2189(63.3)$ & $2204(48.4)$ & & 2427 (48.6) & $1966(78.1)$ & \\
\hline \multirow[t]{2}{*}{ FPG } & Ideal & $2426(79.4)$ & $3513(87.7)$ & $<0.01$ & $4162(88.6)$ & $1777(68.4)$ & $<0.01$ \\
\hline & Non-ideal & $903(20.6)$ & $657(12.3)$ & & $661(11.4)$ & $899(31.6)$ & \\
\hline \multirow[t]{2}{*}{ Age } & $<60$ years & $2078(76.2)$ & 2745 (74.7) & 0.01 & $4823(100.0)$ & $0(0.0)$ & NA \\
\hline & $\geq 60$ years & $1251(23.8)$ & $1425(25.3)$ & & $0(0.0)$ & $2676(100.0)$ & \\
\hline \multirow[t]{2}{*}{ Sex } & Male & $3329(100.0)$ & $0(0.0)$ & NA & $2078(49.8)$ & $1251(47.8)$ & 0.01 \\
\hline & Female & $0(0.0)$ & $4170(100.0)$ & & $2745(50.2)$ & 1425 (52.2) & \\
\hline \multirow[t]{2}{*}{ Education level } & High & $1620(57.6)$ & $2113(58.1)$ & 0.76 & $2969(67.5)$ & $764(28.3)$ & $<0.01$ \\
\hline & Low & 1709 (42.4) & 2057 (41.9) & & $1854(32.5)$ & $1912(71.7)$ & \\
\hline \multirow[t]{2}{*}{ Income } & High & $1642(56.5)$ & 1758 (49.9) & $<0.01$ & $2771(62.0)$ & $629(26.6)$ & $<0.01$ \\
\hline & Low & $1441(43.5)$ & $1976(50.1)$ & & $1628(38.0)$ & $1789(73.4)$ & \\
\hline \multirow[t]{3}{*}{ Region } & $\begin{array}{l}\text { Major } \\
\text { cities }\end{array}$ & $2024(72.0)$ & $2538(73.0)$ & 0.71 & $3012(74.2)$ & $1550(67.2)$ & $<0.01$ \\
\hline & $\begin{array}{l}\text { Inner } \\
\text { regional }\end{array}$ & $760(20.0)$ & $949(19.2)$ & & 1025 (18.3) & $684(23.7)$ & \\
\hline & Other & $545(8.0)$ & $683(7.8)$ & & $786(7.5)$ & $442(9.1)$ & \\
\hline
\end{tabular}

Numbers and percentages were expressed as weighted and unweighted and $p$ values were from weighted chi-square tests. Abbreviations: IHD, ischemic heart disease; BMI, body mass index; TC, total cholesterol; BP, blood pressure; FPG, fasting plasma glucose; NA, not applicable. 


\subsection{Sex and Age-Specific Effects of Individual Metrics on IHD Prevalence}

Tables 2 and 3 list the sex and age-specific crude and adjusted IRRs and 95\% CIs by CVH metric for IHD prevalence, respectively. After adjusted for covariates, physical inactivity (IRR: 1.84, 95\% CI: $1.00-3.39, p=0.048$ ) and elevated TC values (IRR: $1.53,95 \%$ CI: $1.04-2.25, p=0.03$ ) were significant IHD risk factors for males. None of the metrics was associated with IHD prevalence for females. For the analyses of interaction terms, we failed to detect the sexual differences in the associations between those metrics and IHD prevalence ( $P_{\text {interaction }}>0.05$ ). Physical inactivity (IRR: $1.63,95 \%$ CI: $1.01-2.64$, $p=0.046$ ) and elevated TC (IRR: 1.67, 95\% CI: 1.11-2.51, $p=0.02$ ) were significant IHD risk factors for older adults. None of the metrics was associated with IHD prevalence for young adults. The effects of high BMI $\left(P_{\text {interaction }}=0.01\right)$, physical inactivity $\left(P_{\text {interaction }}=0.03\right)$, elevated BP $\left(P_{\text {interaction }}<0.01\right)$, and elevated FPG $\left(P_{\text {interaction }}=0.04\right)$ were more pronounced among young adults than in older adults.

Table 2. IRRs between individual CVH metrics and IHD prevalence, stratified by sex.

\begin{tabular}{ccccccc}
\hline Metrics & Population & $\begin{array}{c}\text { Crude IRR } \\
\mathbf{( 9 5 \%} \text { CI) }\end{array}$ & $p$ & $\begin{array}{c}\text { Adjusted IRR * } \\
\mathbf{( 9 5 \% ~ C I ) ~}\end{array}$ & $p$ & $\boldsymbol{P}_{\text {interaction }}$ \\
\hline Smoking & Males & $1.84(1.21-2.79)$ & 0.01 & $1.12(0.77-1.63)$ & 0.56 & 0.49 \\
& Females & $1.30(0.78-2.18)$ & 0.31 & $1.41(0.79-2.51)$ & 0.24 & \\
\hline \multirow{2}{*}{ High BMI } & Males & $1.93(1.26-2.97)$ & $<0.01$ & $1.32(0.91-1.90)$ & 0.14 & 0.14 \\
& Females & $2.13(1.27-3.58)$ & 0.01 & $1.51(0.85-2.71)$ & 0.15 & \\
\hline Physical inactivity & Males & $3.57(1.94-6.55)$ & $<0.01$ & $1.84(1.00-3.39)$ & 0.048 & 0.38 \\
& Females & $9.47(1.88-47.73)$ & 0.01 & $3.99(0.79-20.12)$ & 0.09 & \\
\hline Unhealthy dietary pattern & Males & $0.42(0.17-1.06)$ & 0.06 & $1.13(0.52-2.43)$ & 0.76 & 0.82 \\
& Females & $1.24(0.50-3.07)$ & 0.64 & $1.33(0.53-3.29)$ & 0.54 & \\
\hline Elevated TC & Males & $2.56(1.66-3.95)$ & $<0.01$ & $1.53(1.04-2.25)$ & 0.03 & 0.85 \\
& Females & $5.22(2.07-13.18)$ & $<0.01$ & $1.70(0.65-4.42)$ & 0.28 & \\
\hline Elevated BP & Males & $1.62(1.07-2.45)$ & 0.02 & $0.81(0.57-1.16)$ & 0.25 & 0.89 \\
& Females & $3.46(1.93-6.21)$ & $<0.01$ & $0.85(0.43-1.69)$ & 0.63 & \\
\hline Elevated FPG & Males & $2.56(1.84-3.55)$ & $<0.01$ & $1.20(0.85-1.67)$ & 0.29 & 1.00 \\
& Females & $2.98(1.66-5.34)$ & $<0.01$ & $1.20(0.56-2.57)$ & 0.64 & \\
\hline
\end{tabular}

* Adjusted for age, education attainment, income, and residence region. Abbreviations: IRR, incidence rate ratio; CI, confidence interval; BMI, body mass index; TC, total cholesterol; BP, blood pressure; FPG, fasting plasma glucose.

Table 3. IRRs between individual CVH metrics and IHD prevalence, stratified by age.

\begin{tabular}{|c|c|c|c|c|c|c|}
\hline Metrics & Population & $\begin{array}{c}\text { Crude IRR } \\
(95 \% \mathrm{CI})\end{array}$ & $p$ & $\begin{array}{l}\text { Adjusted IRR * } \\
(95 \% \mathrm{CI})\end{array}$ & $p$ & $P_{\text {interaction }}$ \\
\hline \multirow[t]{2}{*}{ Smoking } & Young adults & $1.67(0.89-3.14)$ & 0.11 & $1.10(0.55-2.18)$ & 0.78 & 0.48 \\
\hline & Older adults & $1.35(0.95-1.92)$ & 0.09 & $1.22(0.84-1.75)$ & 0.29 & \\
\hline \multirow[t]{2}{*}{ High BMI } & Young adults & $4.42(1.64-11.92)$ & $<0.01$ & $2.28(0.84-6.16)$ & 0.10 & 0.01 \\
\hline & Older adults & $1.07(0.78-1.47)$ & 0.66 & $1.15(0.86-1.52)$ & 0.34 & \\
\hline \multirow[t]{2}{*}{ Physical inactivity } & Young adults & $7.08(1.56-32.16)$ & 0.01 & $4.34(0.87-21.55)$ & 0.07 & 0.03 \\
\hline & Older adults & $1.91(1.17-3.12)$ & 0.01 & $1.63(1.01-2.64)$ & 0.046 & \\
\hline \multirow[t]{2}{*}{ Unhealthy dietary pattern } & Young adults & $0.93(0.21-4.11)$ & 0.93 & $0.91(0.21-3.95)$ & 0.90 & 0.31 \\
\hline & Older adults & $1.27(0.66-2.44)$ & 0.47 & $1.26(0.67-2.37)$ & 0.47 & \\
\hline \multirow[t]{2}{*}{ Elevated TC } & Young adults & $2.69(1.27-5.66)$ & 0.01 & $1.12(0.51-2.48)$ & 0.77 & 0.51 \\
\hline & Older adults & $1.47(0.98-2.21)$ & 0.06 & $1.67(1.11-2.51)$ & 0.02 & \\
\hline \multirow[t]{2}{*}{ Elevated BP } & Young adults & $3.69(1.92-7.12)$ & $<0.01$ & $1.56(0.67-3.62)$ & 0.29 & $<0.01$ \\
\hline & Older adults & $0.78(0.50-1.22)$ & 0.28 & $0.61(0.40-0.92)$ & 0.02 & \\
\hline \multirow[t]{2}{*}{ Elevated FPG } & Young adults & $3.60(1.77-7.30)$ & $<0.01$ & $1.72(0.77-3.83)$ & 0.18 & 0.04 \\
\hline & Older adults & $1.29(0.92-1.80)$ & 0.14 & $1.05(0.74-1.47)$ & 0.79 & \\
\hline
\end{tabular}

* Adjusted for age, sex, education attainment, income, and residence region. Abbreviations: IRR, incidence rate ratio;

$\mathrm{CI}$, confidence interval; BMI, body mass index; TC, total cholesterol; BP, blood pressure; FPG, fasting plasma glucose.

The sex and age-specific adjusted PAFs were displayed in Figures 1 and 2, respectively. Among males, physical inactivity and elevated TC contributed to $37.6 \%$ and $24.8 \%$ of IHD burden (Figure 1). Elevated TC and physical inactivity contributed to $34.4 \%$ and $34.3 \%$ of IHD burden in older adults (Figure 2). None of the seven metrics was significant contributor of IHD in females and younger adults. 


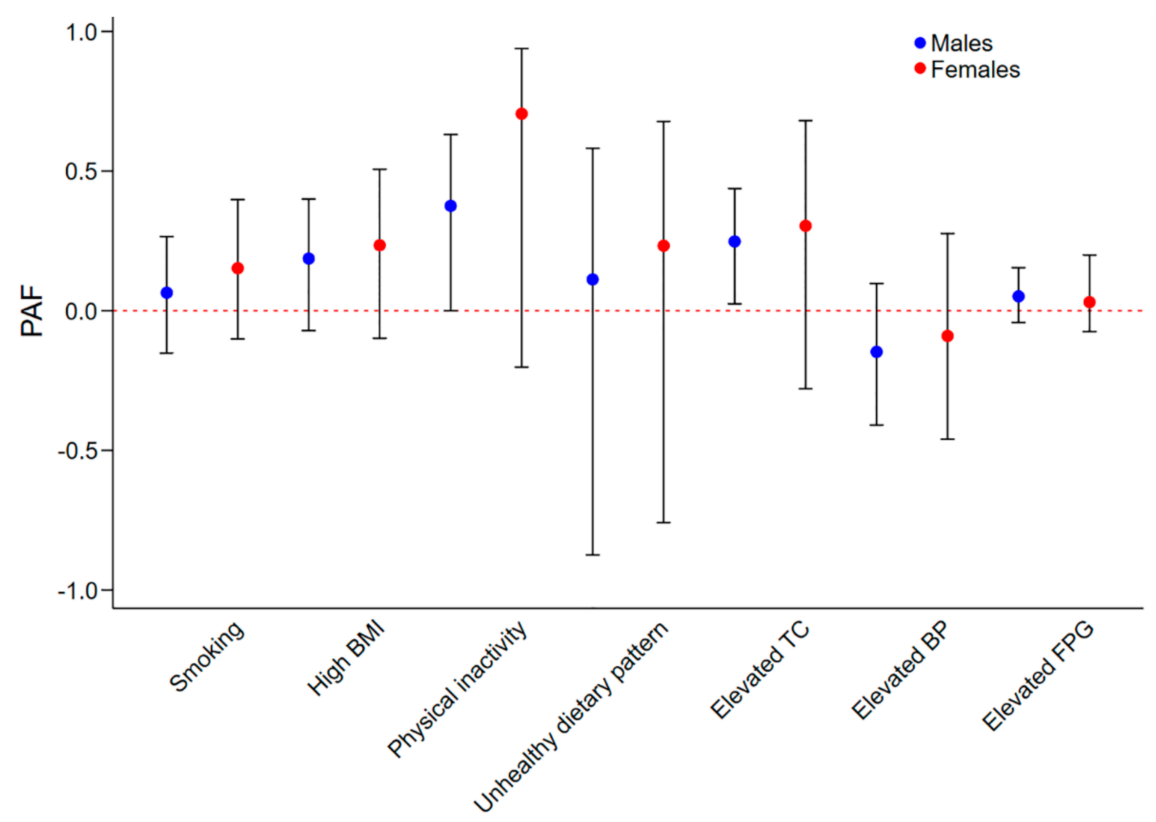

Figure 1. Adjusted PAFs of CVH metrics to IHD prevalence, stratified by sex. Abbreviations: PAF, population attributable fraction; TC, total cholesterol; BP, blood pressure; FPG, fasting plasma glucose; $\mathrm{CVH}$, cardiovascular health; IHD, ischemic heart disease.

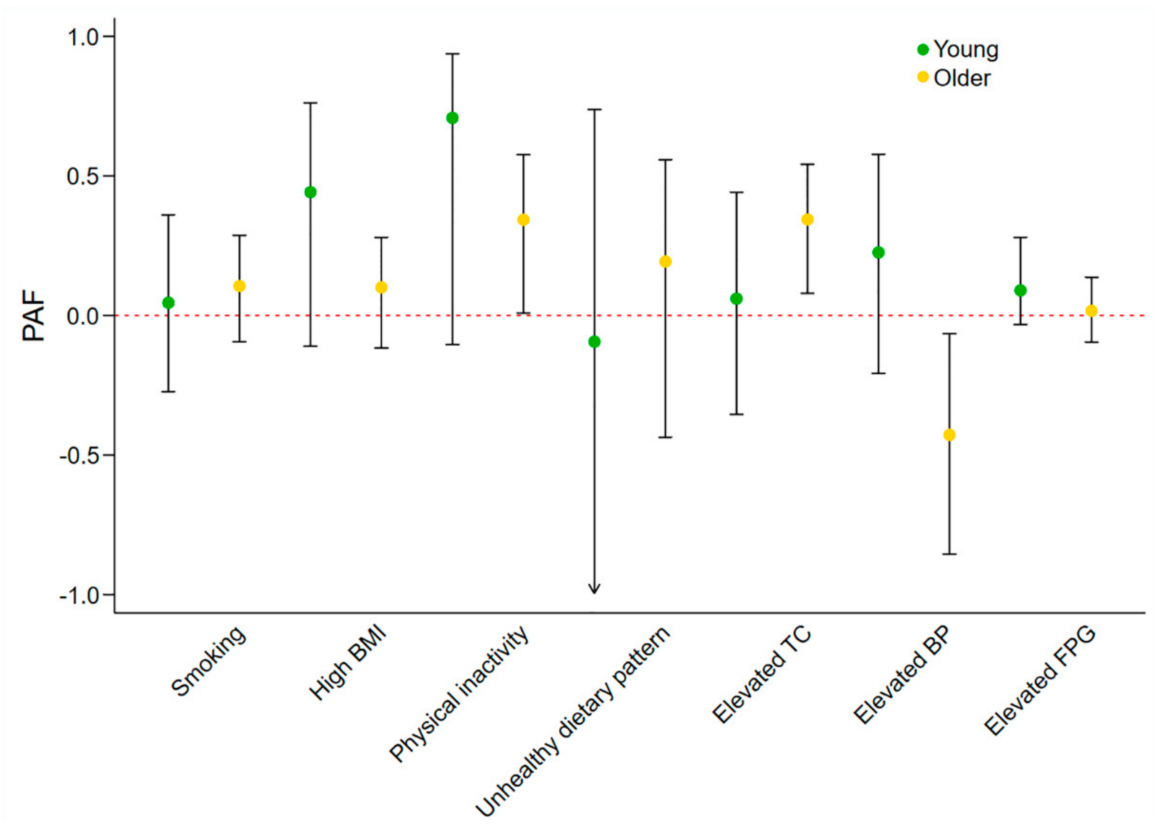

Figure 2. Adjusted PAFs of $\mathrm{CVH}$ metrics to IHD prevalence, stratified by age. Abbreviations: PAF, population attributable fraction; TC, total cholesterol; BP, blood pressure; FPG, fasting plasma glucose; $\mathrm{CVH}$, cardiovascular health; IHD, ischemic heart disease.

\subsection{Sex and Age-Specific Effects of Number of Ideal CVH Metrics on IHD Prevalence}

We explored the association between number of ideal CVH metrics and IHD prevalence based on 7002 participants who have no missing data on the seven metrics and the crude and adjusted ORs were shown in Table 4. We observed a negative association between the number of ideal CVH metrics and IHD prevalence in all sex and age subpopulations. Among females and young adults, we noticed a $59 \%(\mathrm{OR}, 0.41 ; 95 \% \mathrm{CI}, 0.19-0.88)$ and a $65 \%(\mathrm{OR}, 0.35 ; 95 \% \mathrm{CI}, 0.15-0.83)$ reductions in the odds of IHD prevalence for those with intermediate $\mathrm{CVH}$ status and none of those with ideal $\mathrm{CVH}$ status had 
self-reported IHD. For males and older adults, we observed non-significant reductions in the odds of IHD prevalence for those with intermediate and ideal $\mathrm{CVH}$. In addition, one more ideal $\mathrm{CVH}$ metric was associated with $11-31 \%$ reduced IHD prevalence while the association failed to reach significant level for males and older adults. The analyses of interaction terms indicated that the effects of overall $\mathrm{CVH}$ status on IHD prevalence were similar among the four subgroups.

Table 4. ORs between number of ideal metrics and IHD prevalence, stratified by sex and age.

\begin{tabular}{|c|c|c|c|c|c|c|}
\hline Participants & $\begin{array}{l}\text { Ideal Metrics } \\
\text { Number }\end{array}$ & $\begin{array}{l}\text { IHD Cases/ } \\
\text { Participants }\end{array}$ & $\begin{array}{c}\text { Crude OR } \\
(95 \% \mathrm{CI})\end{array}$ & $p$ & $\begin{array}{l}\text { Adjusted * OR } \\
(95 \% \mathrm{CI})\end{array}$ & $p$ \\
\hline \multirow[t]{4}{*}{ Males } & $0-2$ & $150 / 1741$ & Reference & NA & Reference & NA \\
\hline & $3-4$ & $44 / 1147$ & $0.34(0.21-0.56)$ & $<0.01$ & $0.65(0.42-1.01)$ & 0.06 \\
\hline & $5-7$ & $4 / 285$ & $0.06(0.01-0.51)$ & 0.01 & $0.47(0.05-4.30)$ & 0.50 \\
\hline & One more ideal metric & NA & $0.61(0.53-0.70)$ & $<0.01$ & $0.86(0.73-1.02)$ & 0.09 \\
\hline \multirow[t]{4}{*}{ Females } & $0-2$ & $99 / 1601$ & Reference & NA & Reference & NA \\
\hline & $3-4$ & $29 / 1639$ & $0.22(0.11-0.45)$ & $<0.01$ & $0.41(0.19-0.88)$ & 0.02 \\
\hline & $5-7$ & $0 / 589$ & NA & NA & NA & NA \\
\hline & One more ideal metric & NA & $0.52(0.45-0.60)$ & $<0.01$ & $0.73(0.59-0.91)$ & 0.01 \\
\hline \multirow[t]{4}{*}{ Young } & $0-2$ & $54 / 1708$ & Reference & NA & Reference & NA \\
\hline & $3-4$ & $15 / 2028$ & $0.18(0.09-0.37)$ & $<0.01$ & $0.35(0.15-0.83)$ & 0.02 \\
\hline & $5-7$ & $0 / 792$ & NA & NA & NA & NA \\
\hline & One more ideal metric & NA & $0.49(0.40-0.60)$ & $<0.01$ & $0.69(0.52-0.91)$ & 0.01 \\
\hline \multirow[t]{4}{*}{ Older } & $0-2$ & $195 / 1634$ & Reference & NA & Reference & NA \\
\hline & $3-4$ & $58 / 758$ & $0.61(0.39-0.95)$ & 0.03 & $0.68(0.44-1.03)$ & 0.07 \\
\hline & $5-7$ & $4 / 82$ & $0.35(0.04-2.91)$ & 0.32 & $0.55(0.06-5.09)$ & 0.59 \\
\hline & One more ideal metric & $\mathrm{NA}$ & $0.83(0.71-0.97)$ & 0.02 & $0.89(0.76-1.05)$ & 0.17 \\
\hline
\end{tabular}

* Adjusted for age, education attainment, income, and residence region for sex-specific population and additionally adjusted for sex for age-specific population. Abbreviations: OR, odds ratio; IHD, ischemic heart disease; CI, confidence interval; NA, not applicable.

\section{Discussion}

By analyzing the nationally representative survey, we did not reveal the sexual variations in the strength of seven metrics and IHD prevalence. Our results demonstrated that the impacts of high BMI, physical inactivity, elevated BP, and elevated FPG were more apparent in young adults than in older adults. The association between ideal metrics number and IHD prevalence are similar among the four subgroups.

Although we found physical inactivity and elevated TC were significant IHD risk factors and contributors for only males, we did not reveal the differences in the IHD association strengths for all the metrics between men and women. However, a few recent meta-analyses have suggested the possible sex disparities in the effect sizes between CVH metrics and IHD [10-12]. It appears that smoking and diabetes have a more potent influence on CHD risk in women $[10,11]$ whereas elevated TC has more adverse effect in men [12]. We observed that physical inactivity and elevated TC were significant IHD risk factors for older adults instead of young adults. While the findings of interaction terms did not further support the possible age variations. Instead, the interaction term analyses implied the increased BMI, physical inactivity, elevated BP and elevated FPG are associated with greater prevalence in young adults than older adults are. To our knowledge, no meta-analysis explored the age disparities between $\mathrm{CVH}$ metrics and IHD risk. The decreasing effects of high BMI [13], elevated BP [13,14], and elevated FPG $[13,15]$ with age were also noticed in a few observational studies. The age variations in the physical inactivity-IHD relationship were unclear. However, a study has noted the association between physical inactivity and overall CVD was stronger among young adults [16]. Given the inconclusive findings, more large-scale prospective studies are warranted.

We observed that increasing number of ideal metrics was associated with reduced IHD prevalence and the negative association was also found in other studies [2,6]. The very few IHD cases in those with ideal CVH status, especially in females and young adults, suggested the apparent protection effect of $\mathrm{CVH}$ status. The strengths of association was similar among the four groups, which indicate the 
disparities in IHD prevalence was less likely to be explained by the CVH metrics. A number of other factors, including education [17], income [18], and knowledge/awareness of IHD [19] were found to be associated with IHD prevalence and whether they could explain the sex and age disparities in IHD prevalence still need further explorations.

Our study has several strengths: we firstly explored sex and age variances in the associations between CVH metrics and IHD prevalence in Australian adults; we drawn our conclusions based on a nationally representative sample; we adjusted some factors that could be potential confounders. Our study also has several limitations. Firstly, the IHD status was self-reported and it may lead to overestimation or underestimation of IHD prevalence. Previous studies have explored the accuracy of self-reported myocardial infarction, a common type of IHD, and they observed moderate [20,21] or substantial $[22,23]$ agreement between self-reported questionnaire and medical record data. We need studies with medical record diagnosed IHD to focus on the topic. Secondly, demographic factors, smoking, physical activity, and dietary pattern were self-reported, and they may not be wholly accurate. Thirdly, we could not explore the sex and age variations in the associations between CVH metrics and IHD incidence or mortality as our study was a cross-sectional study. Fourthly, we may still suffer from the selection bias even though, as recommended by the ABS, we applied biomedical weights.

\section{Conclusions}

In summary, we noticed the effects of high BMI, physical inactivity, elevated BP, and elevated FPG on IHD prevalence were greater in young adults relative to that in older adults. No sex differences were observed between individual CVH metrics and IHD prevalence. The impacts of ideal metrics number on IHD prevalence were similar among the four subgroups. Further large-scale prospective studies are warranted to confirm the possible sex and age disparities.

Author Contributions: Y.P. and Z.W. contributed to the conception, design, acquisition, analysis, and interpretation of the work. Y.P. drafted the manuscript. Z.W. critically revised the manuscript. All authors gave final approval for the manuscript.

Funding: This research received no external funding.

Conflicts of Interest: The authors declare no conflict of interest.

\section{References}

1. GBD 2016 Causes of Death Collaborators. Global, regional, and national age-sex specific mortality for 264 causes of death, 1980-2016: A systematic analysis for the Global Burden of Disease Study 2016. Lancet 2017, 390, 1151-1210. [CrossRef]

2. Lv, J.; Yu, C.; Guo, Y.; Bian, Z.; Yang, L.; Chen, Y.; Tang, X.; Zhang, W.; Qian, Y.; Huang, Y.; et al. Adherence to Healthy Lifestyle and Cardiovascular Diseases in the Chinese Population. J. Am. Coll. Cardiol. 2017, 69, 1116-1125. [CrossRef] [PubMed]

3. Chomistek, A.K.; Chiuve, S.E.; Eliassen, A.H.; Mukamal, K.J.; Willett, W.C.; Rimm, E.B. Healthy lifestyle in the primordial prevention of cardiovascular disease among young women. J. Am. Coll. Cardiol. 2015, 65, 43-51. [CrossRef] [PubMed]

4. Lloyd-Jones, D.M.; Hong, Y.; Labarthe, D.; Mozaffarian, D.; Appel, L.J.; Van Horn, L.; Greenlund, K.; Daniels, S.; Nichol, G.; Tomaselli, G.F.; et al. Defining and setting national goals for cardiovascular health promotion and disease reduction: The American Heart Association's strategic Impact Goal through 2020 and beyond. Circulation 2010, 121, 586-613. [CrossRef] [PubMed]

5. Peng, Y.; Wang, Z.; Dong, B.; Cao, S.; Hu, J.; Adegbija, O. Life's Simple 7 and ischemic heart disease in the general Australian population. PLoS ONE 2017, 12, e0187020. [CrossRef] [PubMed]

6. Yang, Q.; Cogswell, M.E.; Dana Flanders, W.; Hong, Y.; Zhang, Z.; Loustalot, F.; Gillespie, C.; Merritt, R.; $\mathrm{Hu}$, F.B. Trends in cardiovascular health metrics and associations with all-cause and CVD mortality among us adults. JAMA 2012, 307, 1273-1283. [CrossRef] [PubMed] 
7. Hackshaw, A.; Morris, J.K.; Boniface, S.; Tang, J.L.; Milenkovic, D. Low cigarette consumption and risk of coronary heart disease and stroke: Meta-analysis of 141 cohort studies in 55 study reports. BMJ 2018, 360, j5855. [CrossRef] [PubMed]

8. Huxley, R.R.; Hirakawa, Y.; Hussain, M.A.; Aekplakorn, W.; Wang, X.; Peters, S.A.; Mamun, A.; Woodward, M. Age- and Sex-Specific Burden of Cardiovascular Disease Attributable to 5 Major and Modifiable Risk Factors in 10 Asian Countries of the Western Pacific Region. Circ. J. 2015, 79, 1662-1674. [CrossRef] [PubMed]

9. Gaye, B.; Canonico, M.; Perier, M.C.; Samieri, C.; Berr, C.; Dartigues, J.F.; Tzourio, C.; Elbaz, A.; Empana, J.P. Ideal Cardiovascular Health, Mortality, and Vascular Events in Elderly Subjects: The Three-City Study. J. Am. Coll. Cardiol. 2017, 69, 3015-3026. [CrossRef] [PubMed]

10. Huxley, R.; Barzi, F.; Woodward, M. Excess risk of fatal coronary heart disease associated with diabetes in men and women: Meta-analysis of 37 prospective cohort studies. BMJ 2006, 332, 73-78. [CrossRef] [PubMed]

11. Huxley, R.R.; Woodward, M. Cigarette smoking as a risk factor for coronary heart disease in women compared with men: A systematic review and meta-analysis of prospective cohort studies. Lancet 2011, 378, 1297-1305. [CrossRef]

12. Peters, S.A.; Singhateh, Y.; Mackay, D.; Huxley, R.R.; Woodward, M. Total cholesterol as a risk factor for coronary heart disease and stroke in women compared with men: A systematic review and meta-analysis. Atherosclerosis 2016, 248, 123-131. [CrossRef]

13. Singh, G.M.; Danaei, G.; Farzadfar, F.; Stevens, G.A.; Woodward, M.; Wormser, D.; Kaptoge, S.; Whitlock, G.; Qiao, Q.; Lewington, S.; et al. The age-specific quantitative effects of metabolic risk factors on cardiovascular diseases and diabetes: A pooled analysis. PLoS ONE 2013, 8, e65174. [CrossRef] [PubMed]

14. Lacey, B.; Lewington, S.; Clarke, R.; Kong, X.L.; Chen, Y.; Guo, Y.; Yang, L.; Bennett, D.; Bragg, F.; Bian, Z.; et al. Age-specific association between blood pressure and vascular and non-vascular chronic diseases in 0.5 million adults in China: A prospective cohort study. Lancet Glob. Health 2018, 6, e641-e649. [CrossRef]

15. Rao Kondapally Seshasai, S.; Kaptoge, S.; Thompson, A.; Di Angelantonio, E.; Gao, P.; Sarwar, N.; Whincup, P.H.; Mukamal, K.J.; Gillum, R.F.; Holme, I.; et al. Diabetes mellitus, fasting glucose, and risk of cause-specific death. N. Engl. J. Med. 2011, 364, 829-841. [PubMed]

16. Manson, J.E.; Greenland, P.; LaCroix, A.Z.; Stefanick, M.L.; Mouton, C.P.; Oberman, A.; Perri, M.G.; Sheps, D.S.; Pettinger, M.B.; Siscovick, D.S. Walking compared with vigorous exercise for the prevention of cardiovascular events in women. N. Engl. J. Med. 2002, 347, 716-725. [CrossRef] [PubMed]

17. Tillmann, T.; Vaucher, J.; Okbay, A.; Pikhart, H.; Peasey, A.; Kubinova, R.; Pajak, A.; Tamosiunas, A.; Malyutina, S.; Hartwig, F.P.; et al. Education and coronary heart disease: Mendelian randomisation study. BMJ 2017, 358, j3542. [CrossRef] [PubMed]

18. Konttinen, H.; Kilpi, F.; Moustgaard, H.; Martikainen, P. Socioeconomic Position and Antidepressant Use as Predictors of Coronary Heart Disease Mortality: A Population-Based Registry Study of 362,271 Finns. Psychosom. Med. 2016, 78, 144-152. [CrossRef] [PubMed]

19. Ramachandran, H.J.; Wu, V.X.; He, H.G.; Jiang, Y.; Wang, W. Awareness, knowledge, healthy lifestyle behaviors, and their correlates to coronary heart disease among working women in Singapore. Heart Lung 2016, 45, 341-349. [CrossRef] [PubMed]

20. Yasaitis, L.C.; Berkman, L.F.; Chandra, A. Comparison of self-reported and Medicare claims-identified acute myocardial infarction. Circulation 2015, 131, 1477-1485. [CrossRef] [PubMed]

21. Muggah, E.; Graves, E.; Bennett, C.; Manuel, D.G. Ascertainment of chronic diseases using population health data: A comparison of health administrative data and patient self-report. BMC Public Health 2013, 13, 16. [CrossRef] [PubMed]

22. Okura, Y.; Urban, L.H.; Mahoney, D.W.; Jacobsen, S.J.; Rodeheffer, R.J. Agreement between self-report questionnaires and medical record data was substantial for diabetes, hypertension, myocardial infarction and stroke but not for heart failure. J. Clin. Epidemiol. 2004, 57, 1096-1103. [CrossRef] [PubMed]

23. Bolland, M.J.; Barber, A.; Doughty, R.N.; Grey, A.; Gamble, G.; Reid, I.R. Differences between self-reported and verified adverse cardiovascular events in a randomised clinical trial. BMJ Open 2013, 3. [CrossRef] [PubMed]

(C) 2018 by the authors. Licensee MDPI, Basel, Switzerland. This article is an open access article distributed under the terms and conditions of the Creative Commons Attribution (CC BY) license (http://creativecommons.org/licenses/by/4.0/). 